\title{
Cerium oxide nanoparticle treatment ameliorates peritonitis-induced diaphragm dysfunction
}

\author{
This article was published in the following Dove Press journal: \\ International Journal of Nanomedicine \\ 5 October 2015 \\ Number of times this article has been viewed
}

\author{
Shinichi Asano ${ }^{1,2}$ \\ Ravikumar Arvapalli ${ }^{\prime}$ \\ Nandini DPK Manne $e^{1,2}$ \\ Mani Maheshwari',3 \\ Bing Ma ${ }^{1,2}$ \\ Kevin M Rice' \\ Vellaisamy Selvaraj ${ }^{1,2}$ \\ Eric R Blough ${ }^{1-3}$ \\ 'Center for Diagnostic Nanosystems, \\ ${ }^{2}$ Department of Pharmacology, \\ Pharmaceutical Sciences and Research, \\ School of Pharmacy, ${ }^{3}$ Department \\ of Pharmacology, Physiology and \\ Toxicology, Joan C Edwards School \\ of Medicine, Marshall University, \\ Huntington, WV, USA
}

Correspondence: Shinichi Asano Center for Cardiovascular and Respiratory Sciences, School of Medicine, West Virginia University, I Medical Center Drive, PO Box 9227 , Morgantown, WV 26506, USA

Tel +l 3042938878

Fax + I 3042937105

Email sasano@hsc.wvu.edu

\begin{abstract}
The severe inflammation observed during sepsis is thought to cause diaphragm dysfunction, which is associated with poor patient prognosis. Cerium oxide $\left(\mathrm{CeO}_{2}\right)$ nanoparticles have been posited to exhibit anti-inflammatory and antioxidative activities suggesting that these particles may be of potential use for the treatment of inflammatory disorders. To investigate this possibility, Sprague Dawley rats were randomly assigned to the following groups: sham control, $\mathrm{CeO}_{2}$ nanoparticle treatment only $(0.5 \mathrm{mg} / \mathrm{kg}$ iv $)$, sepsis, and sepsis $+\mathrm{CeO}_{2}$ nanoparticles. Sepsis was induced by the introduction of cecal material $(600 \mathrm{mg} / \mathrm{kg})$ directly into the peritoneal cavity. Nanoparticle treatment decreased sepsis-associated impairments in diaphragmatic contractile $\left(P_{\mathrm{o}}\right)$ function (sham: $25.6 \pm 1.6 \mathrm{~N} / \mathrm{cm}^{2}$ vs $\mathrm{CeO}_{2}: 23.4 \pm 0.8 \mathrm{~N} / \mathrm{cm}^{2}$, vs Sep: $15.9 \pm 1.0 \mathrm{~N} / \mathrm{cm}^{2}$ vs Sep $\left.+\mathrm{CeO}_{2}: 20.0 \pm 1.0 \mathrm{~N} / \mathrm{cm}^{2}, P<0.05\right)$. These improvements in diaphragm contractile function were accompanied by a normalization of protein translation signaling (Akt, FOXO-1, and 4EBP1), diminished proteolysis (caspase 8 and ubiquitin levels), and decreased inflammatory signaling (Stat3 and iNOS). Histological analysis suggested that nanoparticle treatment was associated with diminished sarcolemma damage and diminished inflammatory cell infiltration. These data indicate $\mathrm{CeO}_{2}$ nanoparticles may improve diaphragmatic function in the septic laboratory rat.
\end{abstract}

Keywords: cerium oxide nanoparticles, anti-inflammatory, nitric oxide, diaphragm

\section{Introduction}

The pharmacological application of nanotechnology has become an area of intense interest in recent years, and some have posited that cerium oxide $\left(\mathrm{CeO}_{2}\right)$ nanoparticles may be uniquely suited for several different types of biomedical applications. ${ }^{1}$ Ceria is an element of the lanthanide series that when complexed with oxygen allows it to effectively transition between oxidative states $\left(\mathrm{Ce}^{+3}\right.$ and $\left.\mathrm{Ce}^{+4}\right)$ where it can participate in redox cycling and function as antioxidant. ${ }^{2}$ Other data have suggested that $\mathrm{CeO}_{2}$ nanoparticles are thought to be largely nontoxic under physiologic conditions, ${ }^{3}$ that they appear to be well tolerated in vivo, ${ }^{4}$ that may also exhibit antimicrobial effects, ${ }^{5}$ and that they may be useful for the treatment of disease states characterized by elevations in cellular reactive oxygen species. ${ }^{6}$ Consistent with these data, recent studies have demonstrated that $\mathrm{CeO}_{2}$ nanoparticles may be useful for the treatment of conditions associated with elevated oxidative stress including cardiac dysfunction, some types of neurodegeneration, cancer, and inflammation..$^{7-10}$ Despite numerous medical advancements, the mortality rate associated with sepsis-associated multiple organ dysfunction syndrome remains unacceptably high as it is the leading cause of death in the hospital intensive care unit. ${ }^{11}$ Sepsis and other severe inflammatory disorders are often associated with diaphragm dysfunction, which if uncorrected oftentimes results in decreased survivability and the need for ventilator support. ${ }^{12-14}$ 
Recent work by our laboratory has suggested that treatment with $\mathrm{CeO}_{2}$ nanoparticles is associated with diminished sepsisinduced mortality and decreased levels of systemic inflammation after polymicrobial insult. ${ }^{15}$ Whether this increase in survivability following $\mathrm{CeO}_{2}$ nanoparticle intervention also associated with improvements of diaphragm function is currently unclear. On the basis of previous data demonstrating that rodent diaphragm is particularly susceptible to elevations in oxidative stress and circulating cytokines, ${ }^{16-18}$ we hypothesized that septic animals that had been treated with $\mathrm{CeO}_{2}$ nanoparticles would exhibit improved diaphragmatic function compared with that observed in the untreated counterparts. Our findings suggest that $\mathrm{CeO}_{2}$ nanoparticle treatment improves diaphragm contractility following a severe polymicrobial insult, and that this finding is associated with evidence of diminished muscle damage and inflammation.

\section{Materials and methods}

\section{Materials}

All chemicals were purchased from Sigma-Aldrich Co. (St Louis, MO, USA), unless otherwise specifically stated. Antibodies against Phospho-Akt (Ser473) (C31E5E)(\#4060), Akt (pan) (40D4) (\#2920), Phospho-Stat3 (Tyr705) (D3A7) XP (\#9145), Stat3 (\#9132), $\alpha$-tubulin (\#2144), Bax (\#2772), Bcl-2 (\#2876), Ubiquitin (p4D1) (\#3936), 4E-BP1 phos(thr37/46) (\#9459), and 4E-BP1 Non phos (thr46) (\#4923) were purchased from Cell Signaling Technology (Beverly, MA, USA). Horseradish peroxidase-conjugated anti-mouse (\#7076) and anti-rabbit (\#7074) secondary antibodies were also purchased from Cell Signaling Technology. Caspase 8 primary antibody (sc-7890) was obtained from Santa Cruz Biotechnology Inc. (Dallas, TX, USA). iNOS primary antibody (PA1-036) was from Pierce (Rockford, IL, USA).

\section{Animals}

Ten-week-old male Sprague Dawley rats were purchased from Hilltop Laboratories (Scottdale, PA, USA) and housed for 1-2 weeks to allow acclimation before the initiation of any experiments. Animals were housed two to a cage with 12-12 dark-light cycles at $25^{\circ} \mathrm{C}$. Food and water were provided ad libitum. All animal care and experimental procedures were performed after approval by the Marshall University Institutional Animal Care and Use Committee, and in accordance with the Guide for the Care and Use of Laboratory Animals. ${ }^{19}$

\section{Cecal inoculum peritonitis model}

Animals were anesthetized using isoflurane and fecal peritonitis was induced by making a small abdominal incision followed by the introduction of cecal material and closure of the incision as described previously. ${ }^{20}$ The cecal material was prepared by mixing cecal contents obtained from fresh donor rats with $5 \%$ dextrose water to yield a concentration of $600 \mathrm{mg}$ cecal material in $5 \mathrm{~mL} / \mathrm{kg}$. The cecal material was used immediately and prepared fresh for each experiment. The cecal inoculums $(\mathrm{CI})$ and $\mathrm{CI}+\mathrm{CeO}_{2}$ nanoparticle treatment animals received fresh inoculum from the same donor animal for each experiment. Sham control and $\mathrm{CeO}_{2}$-only animals underwent an identical surgery to induce fecal peritonitis but received sterile dextrose water $(5 \mathrm{~mL} / \mathrm{kg}$, ip) only.

\section{$\mathrm{CeO}_{2}$ nanoparticle treatment}

The characterization of $\mathrm{CeO}_{2}$ nanoparticles (US Research Nanomaterials, Inc., Houston, TX, USA) were imaged using a JEOL JEM-2010 (JEOL, Tokyo, Japan) transmission electron microscope as previously described. ${ }^{21,22}$ Scanning transmission electron microscopy images were acquired using an aberration-corrected analytical electron microscope (transmission electron microscope [TEM]/scanning transmission electron microscope [STEM] JEOL JEM-ARM200CF) operated at $200 \mathrm{keV}$.

For the nanoparticle treatment groups, $\mathrm{CeO}_{2}$ nanoparticles $(0.5 \mathrm{mg} / \mathrm{kg})$ in $200 \mu \mathrm{L}$ of sterile distilled water were given via the tail vein at the time of peritonitis induction or sham surgery. This dose was chosen based on our preliminary data from pilot studies examining animal mortality and previously published data. ${ }^{15,21}$ The sham control and the CI groups received vehicle ( $200 \mu \mathrm{L}$ of sterile distilled water). For the biochemical assessments, animals were sacrificed at 3 or 18 hours while functional assessments of diaphragm contractility were performed at 18 hours. These time points were selected based on previous data suggesting that the 3-hour time point appears to represent the initial systemic inflammatory response syndrome phase while the 18-hour time point coincides with the development of multiple organ dysfunction. ${ }^{15}$

\section{In vitro diaphragm contraction}

Diaphragm contractile function was measured as previously described. ${ }^{23,24}$ Briefly, the entire diaphragm with ribs and the central tendon attached was removed from isoflurane anesthetized animals and quickly placed in ice cold Krebs buffer solution containing $(\mathrm{mM}) 118 \mathrm{NaCl}, 4.7 \mathrm{KCl}, 2.5 \mathrm{CaCl}_{2}$, 1.2 $\mathrm{MgSO}_{4}, 10$ glucose, $1.2 \mathrm{NaH}_{2} \mathrm{PO}_{4}$, and $25 \mathrm{NaHCO}_{3}$ oxygenated with $95 \% \mathrm{O}_{2}$ plus $5 \% \mathrm{CO}_{2}$ to maintain $\mathrm{pH} 7.4$. Two rectangular muscle strips approximately 3-4 $\mathrm{mm}$ in width from the costal region of each hemidiaphragm were dissected from the central tendon to the rib along the fiber orientation. 
The central tendon and ribs were tied with silk sutures with loops to mount the muscle strips in the organ bath. The muscle strips were mounted on the two stainless pins in a glass organ bath, and electrical field stimulation (EFS) was delivered using two platinum electrodes along either side of the muscle (Grass Stimulator S88). The Krebs buffer solution in the organ bath contained $10 \mu \mathrm{M}$ d-tubocurarine chloride (Sigma-Aldrich Co.) to block nicotinic acetylcholine receptors during electric stimulations, and the bath temperature was kept at $25^{\circ} \mathrm{C}$. After the 15 -minute equilibration, EFS with 2 milliseconds single square-wave pulse was delivered at super maximal voltage set at $140 \mathrm{~V}$ to contract the muscle strips. To determine optimal tension $\left(L_{\mathrm{o}}\right)$, diaphragm muscle strips were stretched in a stepwise manner, and the singlepulse twitch protocol was repeated to achieve the maximum active tension. Thereafter, tetanic tension was assessed at $L_{\mathrm{o}}$ by measuring active tension developed in response to the sequential stimulation with trains of $1-150 \mathrm{~Hz}$ stimuli (train duration 500 milliseconds). An interval of 3 minutes was used between the muscle contractions. Isometric contraction was recorded using a force displacement transducer (Grass Technologies, Warwick, RI) interfaced with a Grass polygraph, and the data were digitized at $5 \mathrm{kHz}$ using a storage oscilloscope (DSO-8502, Link Instrument Inc., Fairfield, NJ, USA). Specific tension was normalized to the estimated muscle cross-sectional area by the following equation:

Cross sectional area $=$ wet weight $(\mathrm{g}) /$ length $(\mathrm{cm})$ $\times 1.06\left(\right.$ density $\left.\mathrm{g} / \mathrm{cm}^{3}\right){ }^{25}$

\section{Immunoblot analysis}

Semiquantitiative immunodetection was performed as detailed previously. ${ }^{26}$ Briefly, diaphragms were pulverized and then homogenized in Tissue Protein Extraction Reagent (Pierce) supplemented with $1 \mathrm{mM}$ Dithiothreitol (DTT), protease, and phosphatase inhibitor cocktails (Sigma-Aldrich Co.). Lysates were sonicated on ice and centrifuged at $10,000 \times g$ at $4^{\circ} \mathrm{C}$ for 10 minutes. The supernatant was removed, and protein concentration was determined by 660 protein assay reagent (Thermo Fisher Scientific, Waltham, MA, USA). Samples were diluted in Laemmli sample buffer, and equal amounts of protein were separated by SDS-PAGE, followed by electroblotting onto nitrocellulose membranes. Membranes were blocked with $5 \%$ milk in Tris Buffered Saline with Tween (TBST) for 1 hour at room temperature and washed three times in TBST before incubation overnight at $4^{\circ} \mathrm{C}$ with primary antibodies. Membranes were incubated with the secondary antibody conjugated with horseradish peroxidase (HRP) for 1 hour at room temperature. An enhanced chemiluminescence (ECL) was used to detect immunoreactivity using a FluorChem E System (Cell Biosciences, Santa Clara, CA, USA).

\section{Histochemical analysis Procion orange}

A bolus administration of $1.5 \mathrm{~mL}$ (3\% Procion orange in $0.9 \%$ sterile saline) was infused via femoral vein catheter. After allowing the dye to circulate for 15 minutes, the diaphragm muscle was removed and quickly frozen in liquid nitrogencooled isopentane. ${ }^{27}$ In some animals, muscle strips were immersed in HEPES buffer containing $0.2 \%$ Procion orange (Sigma-Aldrich Co.) for 1 hour at room temperature and quickly frozen as described above. ${ }^{28}$ Frozen tissues were serially sectioned in $10 \mu \mathrm{m}$ and placed on poly-lysine-coated slides before imaging with an inverted fluorescent microscope (EVOS AMG, Bothell, WA, USA) under a green fluorescent protein filter (excitation $450 \mathrm{~nm} /$ emission $505 \mathrm{~nm}$ ).

\section{Hematoxylin and eosin staining}

Frozen tissue sections from animals without Procion orange perfusion were fixed in $4 \%$ formalin in phosphate buffer solution (PBS), and the sections were stained with $\mathrm{H} \& \mathrm{E}$ according to the manufacturer (BBC Biochemical, Mount Vernon, WA, USA). Images were obtained using an inverted microscope (EVOS AMG).

\section{Real-time polymerase chain reaction}

Real-time polymerase chain reaction (PCR) was performed for quantification of $i N O S$ and GAPDH using a 7500 Real Time PCR system (Thermo Fisher Scientific, Waltham, MA, USA) as described previously. ${ }^{26}$ Briefly, total RNA was extracted using Trizol reagent (Sigma-Aldrich Co.) according to the manufacturer's guidelines, and cDNA was synthesized using $1 \mu \mathrm{g}$ of RNA with the High-Capacity cDNA Reverse Transcription Kit (Thermo Fisher Scientific). SYBR green PCR amplification was performed with SensiMix ${ }^{\mathrm{TM}}$ SYBR low-ROX kit (Bioline, London, UK) using the following primers: iNOS (NM_012611.3; 5'-GGA AGA GAC GCA CAG GCA GAG-3' and 5'-GCA GGC ACA CGC AAT GAT GG-3'; product size 126 bp) and GAPDH (NM_017008.4; 5'-GTT ACC AGG GCT GCCTTC TC-3' and 5'-GGG TTT CCC GTT GAT GAC C-3'; product size $168 \mathrm{bp}$ ) according to the manufacturer's guidelines. Each sample was analyzed in triplicate in $25 \mu \mathrm{L}$ reactions. A nontemplate control was used as a negative control and included in all PCR reactions. Specific products were determined by the melting curve analysis as well as agarose gel electrophoresis using ethidium bromide. Expression levels between groups were compared using the relative comparison method $\left(2^{-\Delta \Delta \mathrm{CT}}\right)$. 


\section{Statistics}

Data are presented as the mean and standard error of the mean as described in the text and figure legends. Statistical comparisons were made using paired $t$-tests and one- or twoway ANOVA with Bonferroni post hoc tests as appropriate. A $P<0.05$ was considered as significant.

\section{Results \\ $\mathrm{CeO}_{2}$ nanoparticle characterization}

Details regarding the characterization of $\mathrm{CeO}_{2}$ nanoparticles used in this study have been previously published. ${ }^{15}$ SEM and TEM analyses suggested that the particles were likely spherical in shape and $\sim 15-20 \mathrm{~nm}$ in diameter (Figure 1A and B).

\section{$\mathrm{CeO}_{2}$ nanoparticle treatment improves diaphragmatic function in the septic rat}

There were significant differences in pre-/post-body weight and serum hematocrit levels for the $\mathrm{CI}$ and $\mathrm{CI}+\mathrm{CeO}_{2}$ groups but not for the sham and $\mathrm{CeO}_{2}$-only treated animals at 18 hours after the surgery (Table 1). The muscle length at which maximal tension was developed $\left(L_{\mathrm{o}}\right)$ was not different between groups (Table 1). Muscle strips from all groups demonstrated frequency-dependent increased tension (Figure 2A). Peak twitch tension was 93\% $05 \%, 68 \% \pm 4 \%$, and $82 \% \pm 5 \%$ of sham control for $\mathrm{CeO}_{2}$ only, CI, and $\mathrm{CI}+\mathrm{CeO}_{2}$, respectively (Table $1 ; P<0.05$ ). Similarly, peak tetanic tension was $89 \% \pm 3 \%, 62 \% \pm 4 \%$, and $78 \% \pm 4 \%$ of sham control for $\mathrm{CeO}_{2}$ only, $\mathrm{CI}$, and $\mathrm{CI}+\mathrm{CeO}_{2}$, respectively (Figure 2B; $P<0.05$ ). Consistent with these data, sepsis appeared to increase Procion orange dye diffusion into the diaphragm compared with that observed in the other groups (Figure 3A). Histological analysis following H\&E staining suggested that sepsis was associated with increased cell infiltration that appeared to be diminished with nanoparticle treatment (Figure 3B).

\section{$\mathrm{CeO}_{2}$ nanoparticle treatment attenuates sepsis-associated diaphragmatic inflammation}

Nanoparticle treatment decreased sepsis-induced increases in Stat3 phosphorylation at 3- but not 18 hours (Figure 4A; $P<0.05)$. Nanoparticle treatment significantly decreased $i N O S$ transcript levels and protein in the septic animals (Figure 4B and $\mathrm{C} ; P<0.05$ ).

\section{$\mathrm{CeO}_{2}$ nanoparticle treatment suppresses translation-related signaling, caspase 8 cleavage, and protein ubiquination during sepsis}

Compared with the control animals, the phosphorylation of Akt, FOXO-1, and 4EBP-1 was not changed at 3 hours (data not shown). Nanoparticle treatment attenuated sepsisinduced decreases in the phosphorylation of Akt, FOXO-1, and 4EBP-1 at 18 hours (Figure 5A-C; $P<0.05$ ). Similarly, nanoparticle treatment reduced sepsis-associated protein ubiquination at 3 hours (Figure 6A; $P<0.05$ ) and caspase 8 cleavage at 18 hours (Figure 6B; $P<0.05$ ). Neither sepsis nor nanoparticle treatment was associated with changes in caspase 3 cleavage, apoptosis-inducing factor, or the ratio of Bax/Bcl-2 (data not shown).

\section{Discussion}

Previous work from our laboratory using the CI peritonitis model has demonstrated that $\mathrm{CeO}_{2}$ nanoparticle treatment was associated with improved animal survivability and
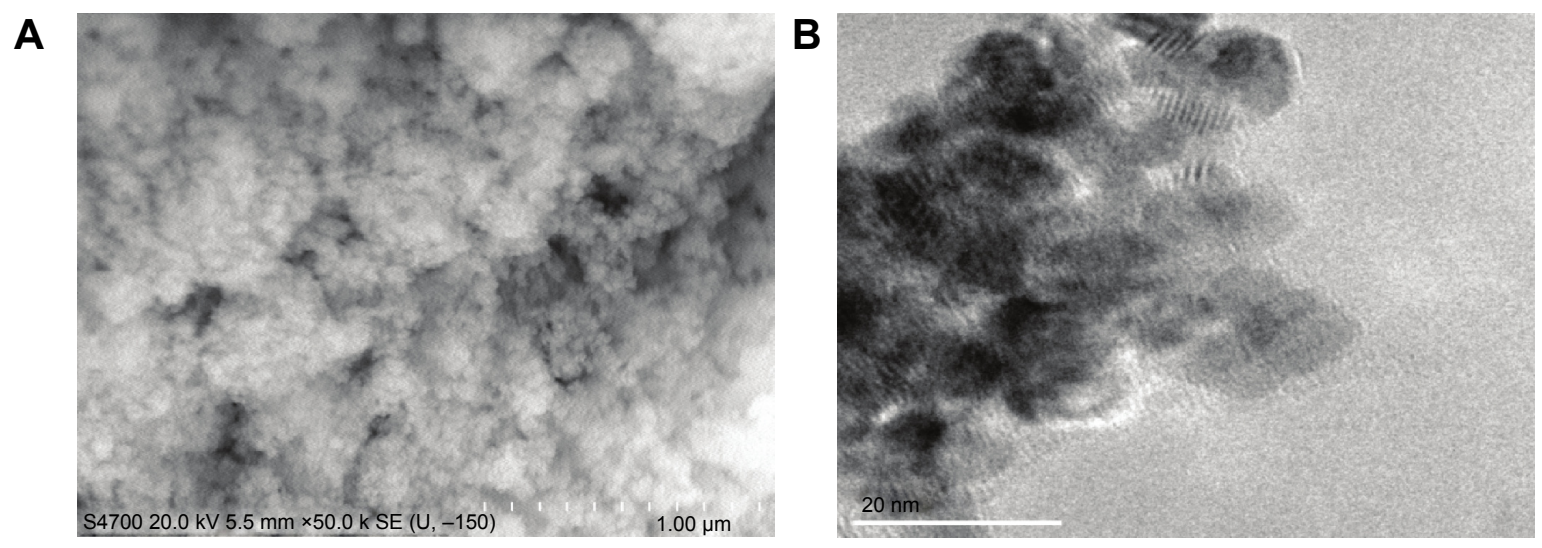

Figure I Characteristics of cerium oxide $\left(\mathrm{CeO}_{2}\right)$ nanoparticles.

Notes: Image of $\mathrm{CeO}_{2}$ nanoparticle powder under transmission electron microscopy is shown (A, scale bar $\left.=\mathrm{I} \mu \mathrm{m}\right)$. CeO ${ }_{2}$ nanoparticles are further characterized by scanning electron microscopy (B, scale bar $=20 \mathrm{~nm})$. 
Table I Characteristics of diaphragm strip and animal each group at 18 hours

\begin{tabular}{|c|c|c|c|c|}
\hline & Sham control & $\mathrm{CeO}_{2}$ only & $\mathbf{C l}$ & $\mathrm{Cl}+\mathrm{CeO}_{2}$ \\
\hline Pre-body weight (g) & $419.3 \pm 1.9$ & $417.9 \pm 8.7$ & $407.3 \pm 5.0$ & $409.6 \pm 2.8$ \\
\hline Post-body weight (g) & $425.3 \pm 2.1$ & $420.8 \pm 8.2$ & $396.1 \pm 4.5^{*}$ & $402.1 \pm 3.5^{*}$ \\
\hline Delta body weight (g) & $6.1 \pm 1.2$ & $2.9 \pm 1.5$ & $-10.0 \pm 1.3^{*}$ & $-7.6 \pm 2.2^{*}$ \\
\hline Hematocrit (\%) & $4 I .3 \pm 4.3$ & $46.0 \pm 4.0$ & $61.8 \pm 3.3 *$ & $58.6 \pm 1.6 *$ \\
\hline Strip weight (mg) & $39.7 \pm 3.7$ & $36.2 \pm 2.8$ & $39.7 \pm 1.7$ & $39.3 \pm 1.9$ \\
\hline$L_{0}(\mathrm{~mm})$ & $20.5 \pm 0.4$ & $20.4 \pm 0.8$ & $20.0 \pm 0.5$ & $19.3 \pm 0.3$ \\
\hline$P_{\mathrm{t}}\left(\mathrm{N} / \mathrm{cm}^{2}\right)$ & $9.68 \pm 0.78$ & $9.04 \pm 0.49$ & $6.56 \pm 0.34 *$ & $7.91 \pm 0.48$ \\
\hline$P_{\mathrm{o}}\left(\mathrm{N} / \mathrm{cm}^{2}\right)$ & $26.45 \pm 1.32$ & $23.68 \pm 0.80$ & $16.4 I \pm 1.03^{*}$ & $20.06 \pm 1.14 * *$ \\
\hline Contraction time (ms) & $51.28 \pm 2.74$ & $55.53 \pm 3.82$ & $49.22 \pm 1.74$ & $48.19 \pm 2.36$ \\
\hline Half relaxation time $(\mathrm{ms})$ & $50.45 \pm 4.00$ & $57.87 \pm 4.58$ & $58.65 \pm 5.27$ & $50.00 \pm 3.14$ \\
\hline
\end{tabular}

Notes: $* P<0.05$ compared with control group. $* * P<0.05$ compared with $\mathrm{Cl}$ group by one-way ANOVA.

Abbreviations: $\mathrm{CeO}_{2}$, cerium oxide; $\mathrm{Cl}$, cecal inoculums; $L_{\mathrm{o}}$, optimal length; $P_{\mathrm{o}}$, maximal tetanic force; $P_{\mathrm{t}}$, maximal tetanic force.

diminished systemic inflammation ${ }^{15}$ with the latter occurring most likely due to diminished Kupffer cell activation. ${ }^{21}$ In this study, we show that $\mathrm{CeO}_{2}$ nanoparticle treatment attenuates the diaphragm dysfunction induced by the septic condition. We believe that these are the first data to demonstrate that nanoparticle treatments designed to target the liver Kupffer cells might be useful for preventing the muscle dysfunction and atrophy seen in clinical conditions characterized by severe systemic inflammation. Similar to earlier studies employing other sepsis models, ${ }^{23,29-31}$ we show that the CI

\section{A Control 18 hours $\quad \mathrm{CeO}_{2} 18$ hours $\quad \mathrm{Cl} 18$ hours $\quad \mathrm{Cl}+\mathrm{CeO}_{2} 18$ hours}
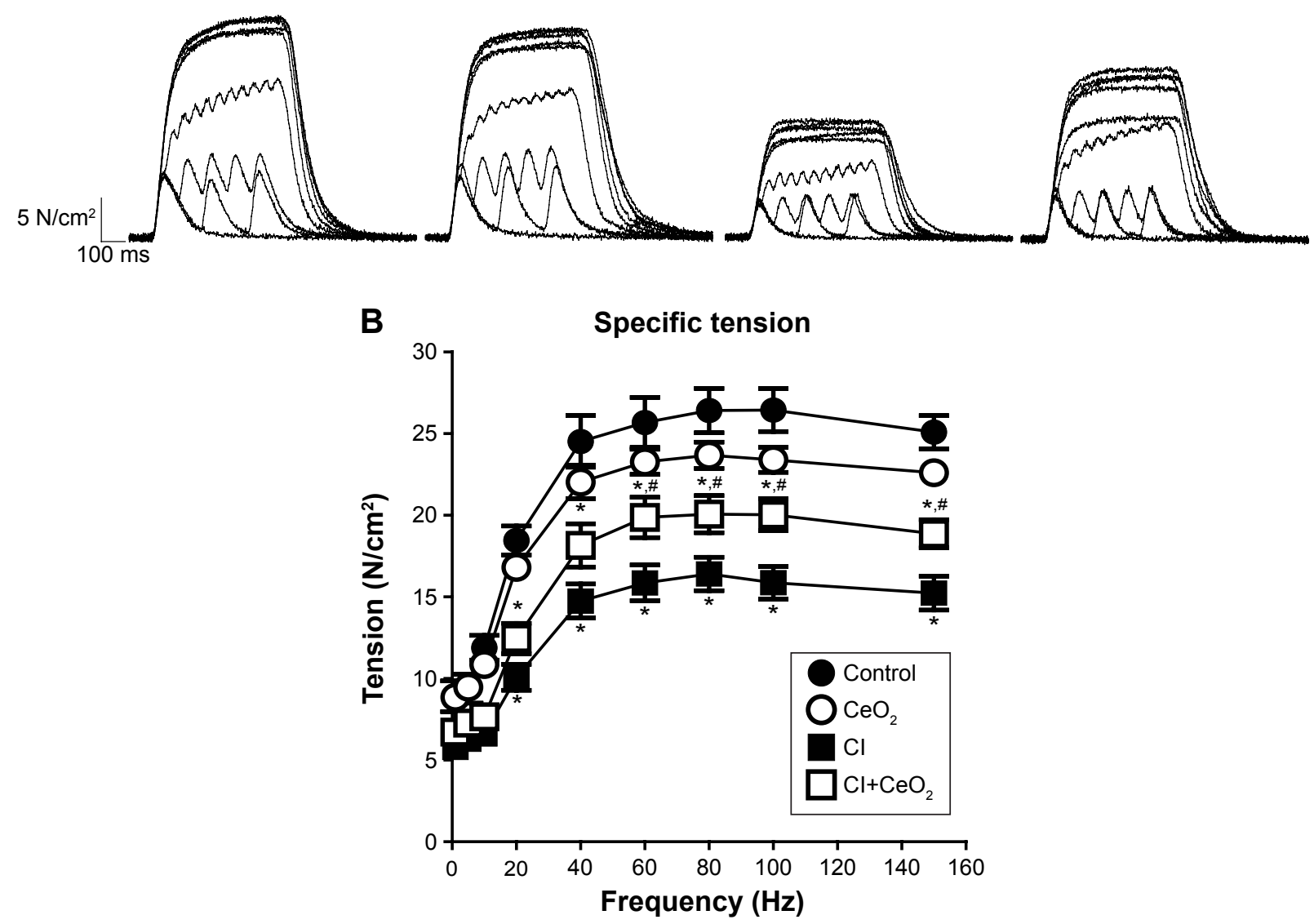

Figure 2 Cerium oxide $\left(\mathrm{CeO}_{2}\right)$ nanoparticle treatment improves diaphragmatic contractility in cecal inoculum $(\mathrm{Cl})$ rat.

Notes: Representative recording traces of contractile response are shown from each group (A). Group data showed that nanoparticle treatment improves diaphragm tension development (B, control $n=8$ strips from 4 rats, $\mathrm{CeO}_{2}$ only $n=6$ strips from 3 rats, cecal inoculum $(\mathrm{Cl}) \mathrm{n}=16$ strips from 8 rats and $\mathrm{Cl}+\mathrm{CeO}{ }_{2} \mathrm{n}=18$ strips from 9 rats). *vs Control, "vs $\mathrm{Cl}: \mathrm{P}<0.05$ by two-way repeated measures ANOVA. 
A
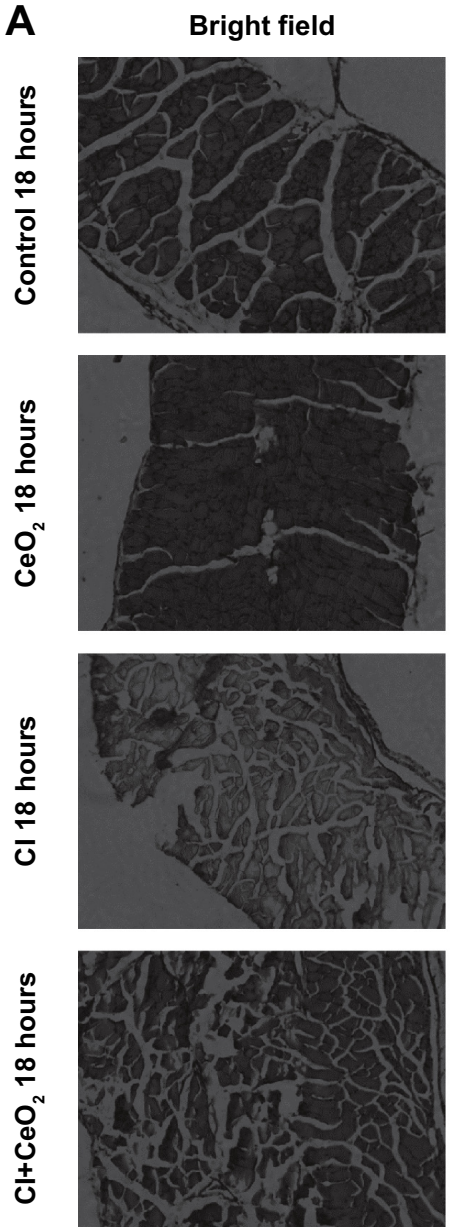

Procion orange
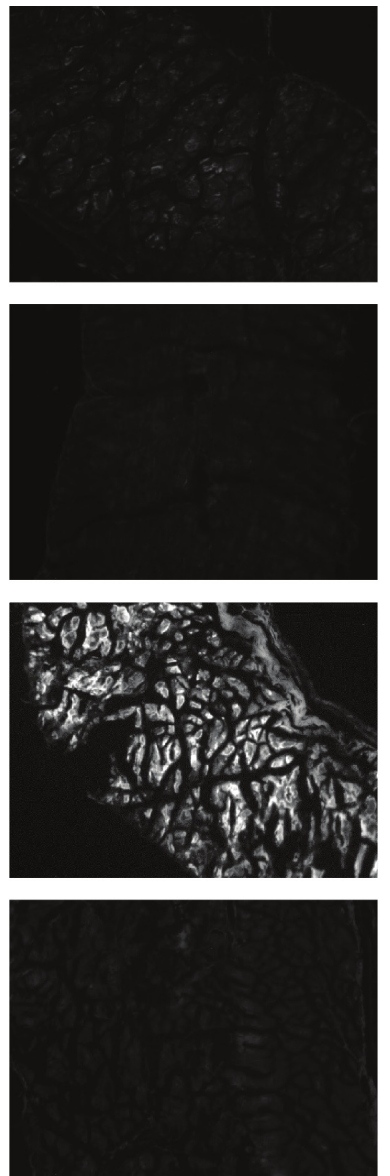

B
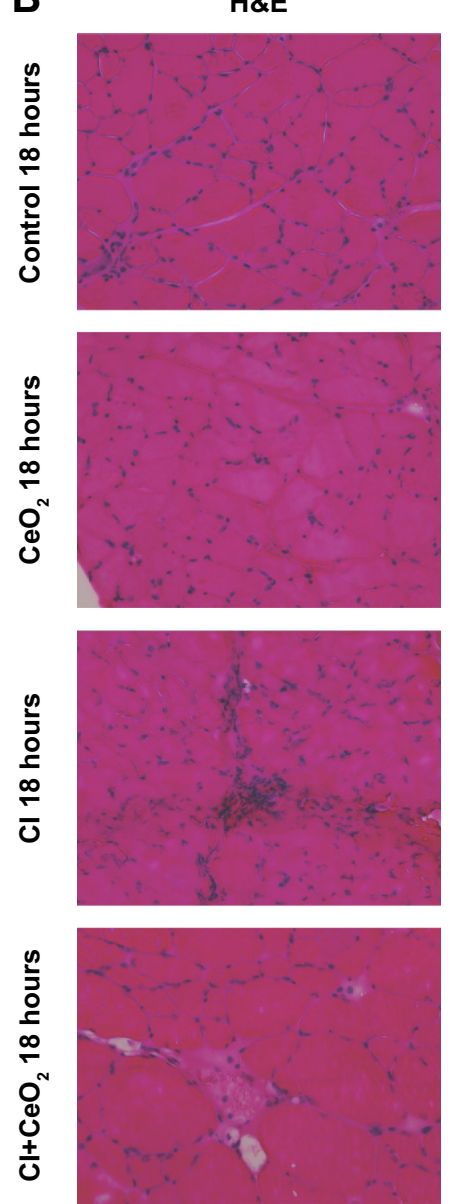

Figure 3 Cerium oxide $\left(\mathrm{CeO}_{2}\right)$ nanoparticle treatment decreases cecal inoculum $(\mathrm{Cl})$ induced muscle damage.

Notes: Nanoparticle treatment functions to protect the muscle membrane as suggested by diminished Procion orange signal in the treated animals. Images were obtained using an inverted fluorescent microscope with I0x objective lens using a GFP filter (A). Sepsis increases and nanoparticle treatment decreases cellular infiltration. Representative bright field images were captured at $20 \times$ objective lens (B).

Abbreviations: GFP, green fluorescent protein; H\&E, hematoxylin and eosin.

peritonitis model is also characterized by alterations in diaphragmatic function (Figure 2). Importantly, we also found that the $\mathrm{CeO}_{2}$ nanoparticle treatment appeared to diminish the effects that the septic condition had on diaphragm tension development.

Whether these improvements in diaphragm contractility also reflect an improved pulmonary function in vivo is currently unclear. Similarly, why muscle function may be decreased with sepsis, or other conditions that are characterized by increased systemic inflammation are not entirely understood; however, it has been suggested that increased cellular infiltration and/or muscle membrane damage may play a role. ${ }^{32}$ To investigate this possibility, we examined the effects of sepsis and nanoparticle treatment on muscle histology and membrane permeability following exposure to Procion dye. Similar to previous reports, ${ }^{32,33}$ we found that the diaphragms obtained from septic animals exhibited a marked increase sarcolemma damage and cellular infiltration (Figure 3A). In addition, and consistent with our findings of improved contractile function with nanoparticle treatment, we found that these changes in cellular structure appeared to be lessened with nanoparticle treatment (Figure 3B). To extend these data, we next examined if the nanoparticle treatment modulated inflammatory signaling in diaphragm. Several studies have suggested that elevations in the local (muscle) or circulating tumor necrosis factor (TNF)-alpha may play a key role in regulating diaphragmatic function during infection. ${ }^{16,23,34-37}$ Our data are consistent with this view and previous work from our laboratory that demonstrated elevations in systemic inflammation in the rat CI peritonitis model. How circulating cytokines cause muscle contractile dysfunction remains unclear; however, it has been shown that the activation of Stat3-iNOS signaling by elevations in IL-6 is associated with diminished cardiac 
A
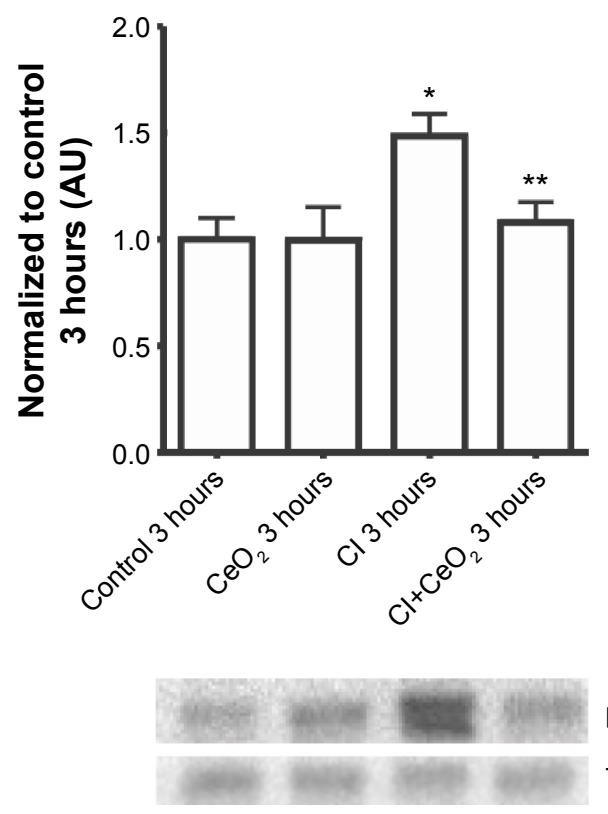

B
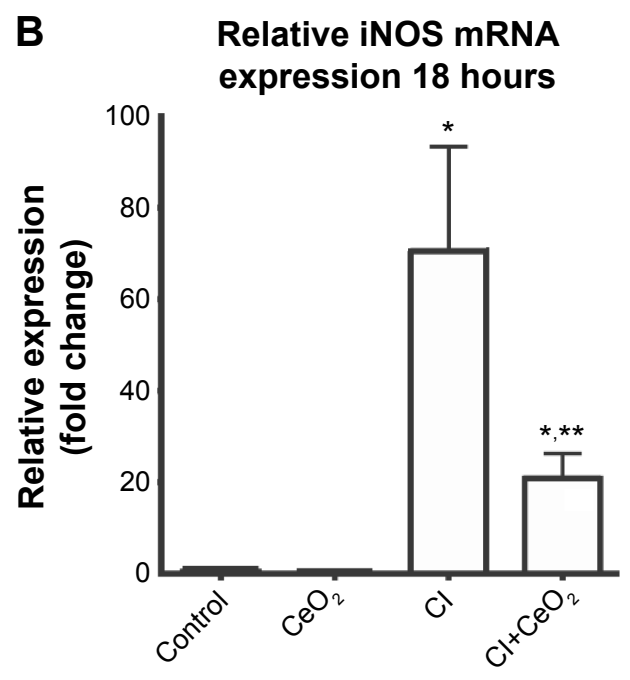

p-Stat3/Stat3 18 hours

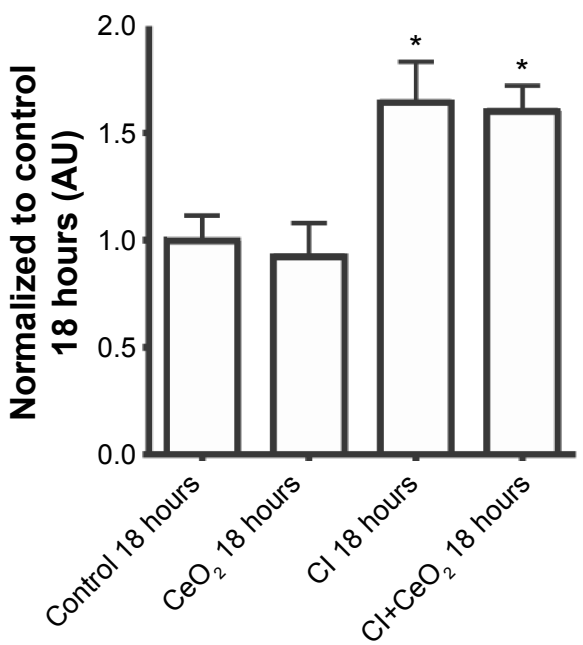

p-Stat3

Total Stat3

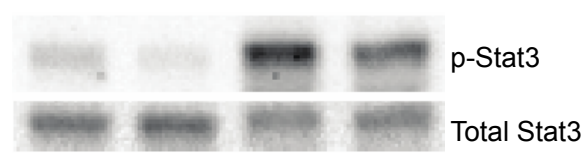

C Protein iNOS expression

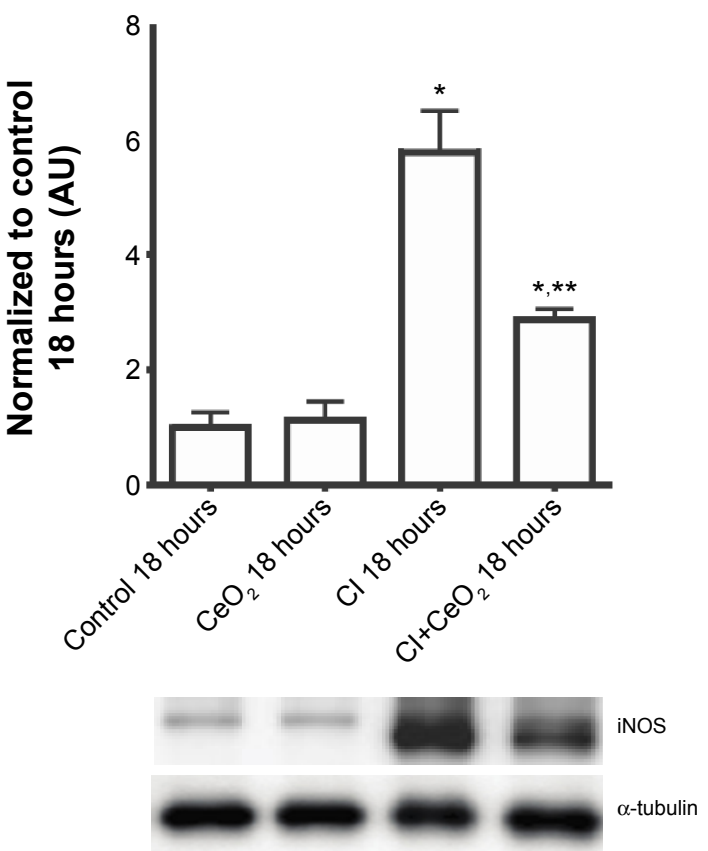

Figure 4 Cerium oxide $\left(\mathrm{CeO}_{2}\right)$ nanoparticle treatment attenuates diaphragmatic inflammation after septic insult.

Notes: Cecal inoculum $(\mathrm{Cl})$ induced increases in Stat 3 phosphorylation are diminished with nanoparticle treatment (A, $\mathrm{n}=6$ rats/each group). Real-time PCR showed that iNOS gene expression levels was increased with $\mathrm{Cl}$ group and diminished with nanoparticle treatment at 18 hours (B, $\mathrm{n}=6 \mathrm{rats} / \mathrm{each}$ group). Immunoblot for iNOS/ $\alpha$-tubulin is shown (C). *vs Control, **vs $\mathrm{Cl}$ : $\mathrm{P}<0.05$ by one-way ANOVA.

Abbreviation: PCR, polymerase chain reaction.

contractility. ${ }^{38}$ Similar to the mechanisms of the inflammatory response in cardiac muscle, we found that sepsis significantly increased Stat3 phosphorylation in diaphragm, and importantly, that this increase was attenuated with nanoparticle treatment (Figure 4A). Likewise, increases in
NO have been shown to directly impair contractile function in a number of different skeletal muscles including the diaphragm..$^{23,30,32,39}$ Consistent with this premise, we found that sepsis significantly increased $i N O S$ gene expression and protein content in diaphragm (Figure 4B and C). Similar 
A

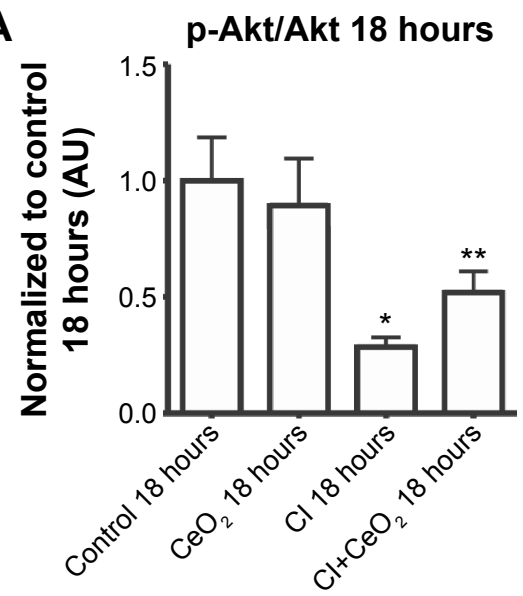

B

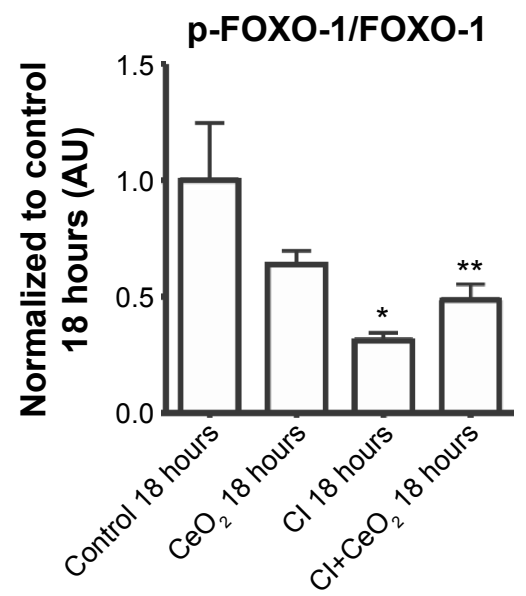

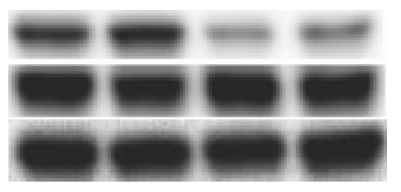

pastuas

Akt alpha

Tubulin

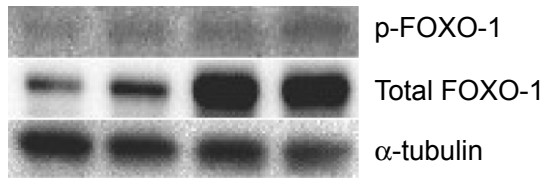

C p-4EBP-1/non-phospho 4EBP-1 18 hours

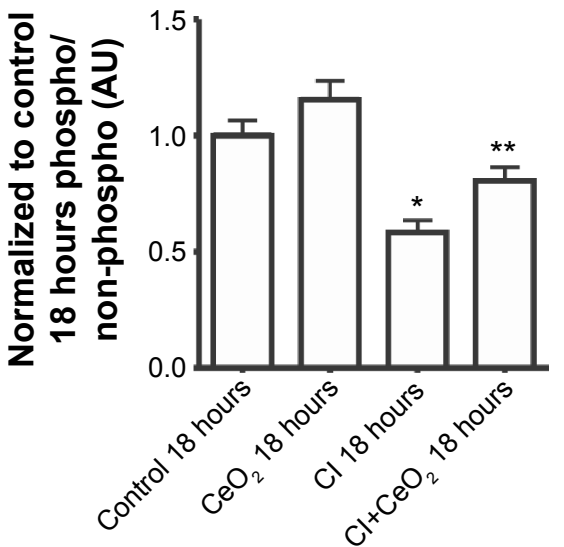

Figure 5 Cerium oxide $\left(\mathrm{CeO}_{2}\right)$ nanoparticle treatment normalizes the disruption of protein synthesis signaling in cecal inoculum $(\mathrm{Cl})$ peritonitis diaphragm. Notes: Effect of nanoparticle on protein synthesis signaling was determined by immunoblot. Nanoparticle treatment increases phospho-Akt (A), phospho-Foxol (B), and phospho-4E-BPI (C) levels in the septic diaphragm ( $n=6$ rats/each group). *vs Control: $P<0.05$ by one-way ANOVA. **vs Cl: $P<0.05$ by $t$-test.

to our contractile measurements, these alterations in NO signaling were attenuated with nanoparticle treatment. Given that $\mathrm{CeO}_{2}$ nanoparticles have been shown to possess not only redox modulation but also immunomodulation properties in vitro, ${ }^{7,8,40}$ it is possible that our in vivo $\mathrm{CeO}_{2}$ nanoparticle treatment effects may be involved in the regulation of immune function and $\mathrm{NO}$-associated oxidative stress. Taken together, these data suggest that nanoparticle treatment attenuated peritonitis-induced diaphragm dysfunction and that this improvement in contractility may be associated with diminished inflammatory signaling.

\section{$\mathrm{CeO}_{2}$ nanoparticle treatment improves protein degradation/ synthesis signaling in the septic diaphragm}

In addition to muscle damage and increased inflammatory signaling, it is also thought that increases in protein degradation 
A
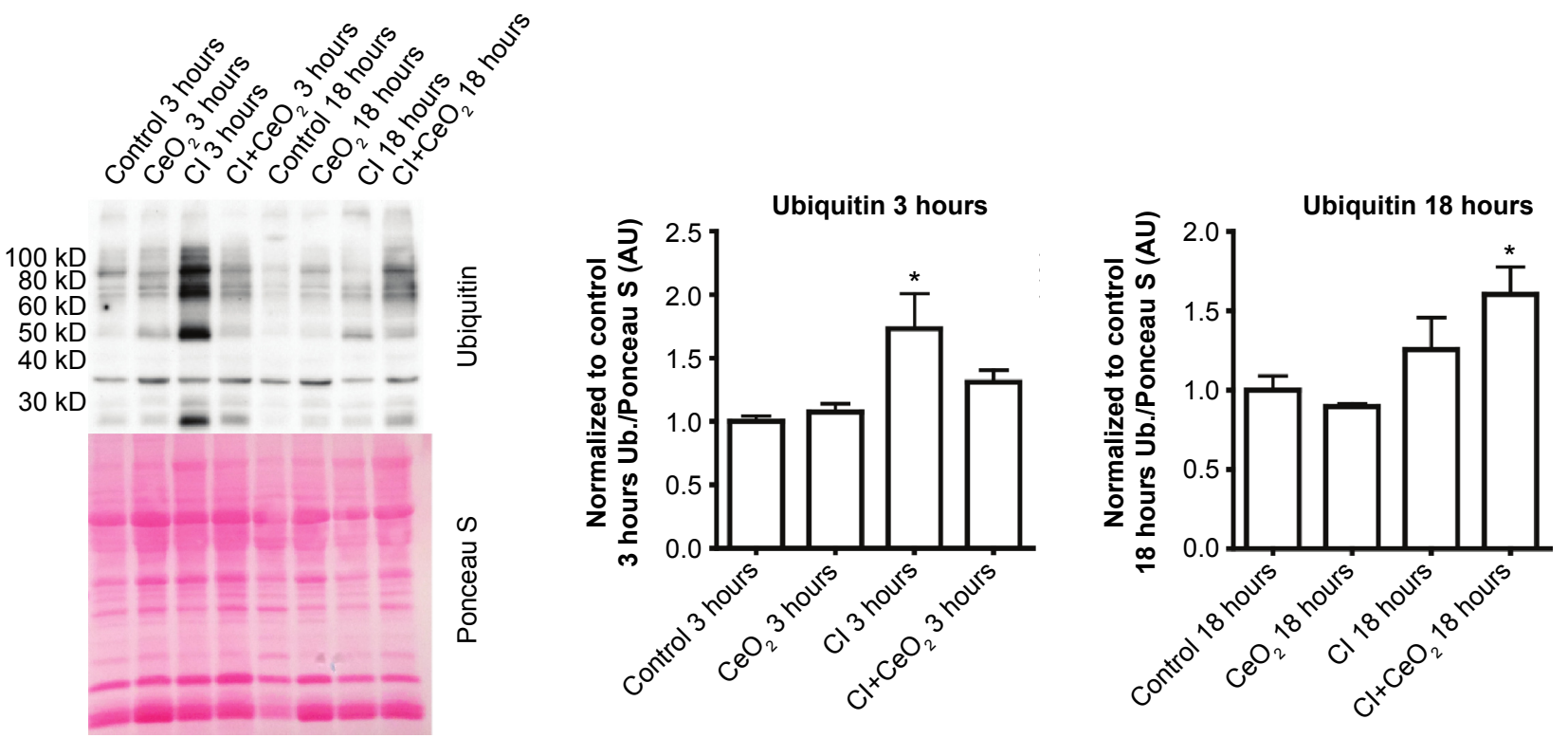

B
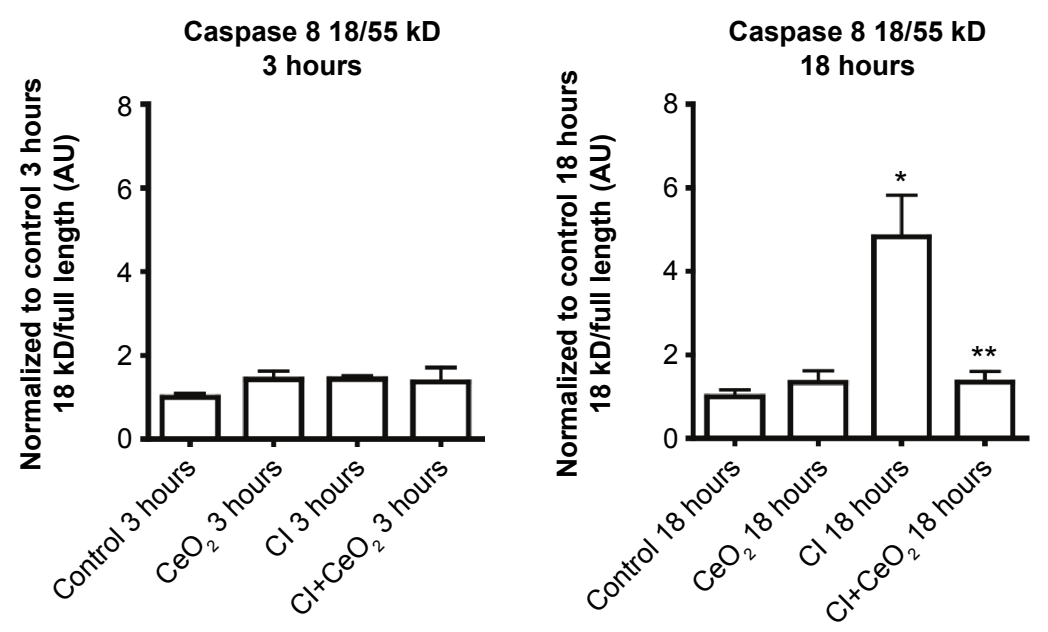

$30 \mathrm{kD}$

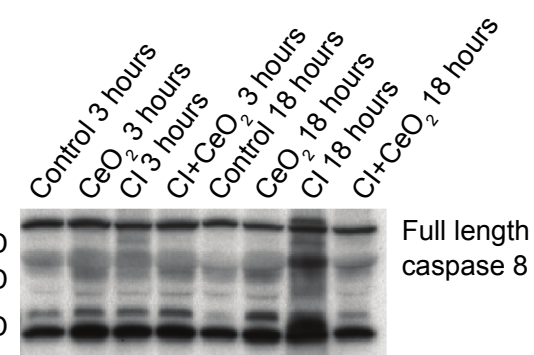

$20 \mathrm{kD}$

Caspase 8

p18

Caspase 8 10/55 kD

3 hours

Caspase 8 10/55 kD

Caspase 8 p10
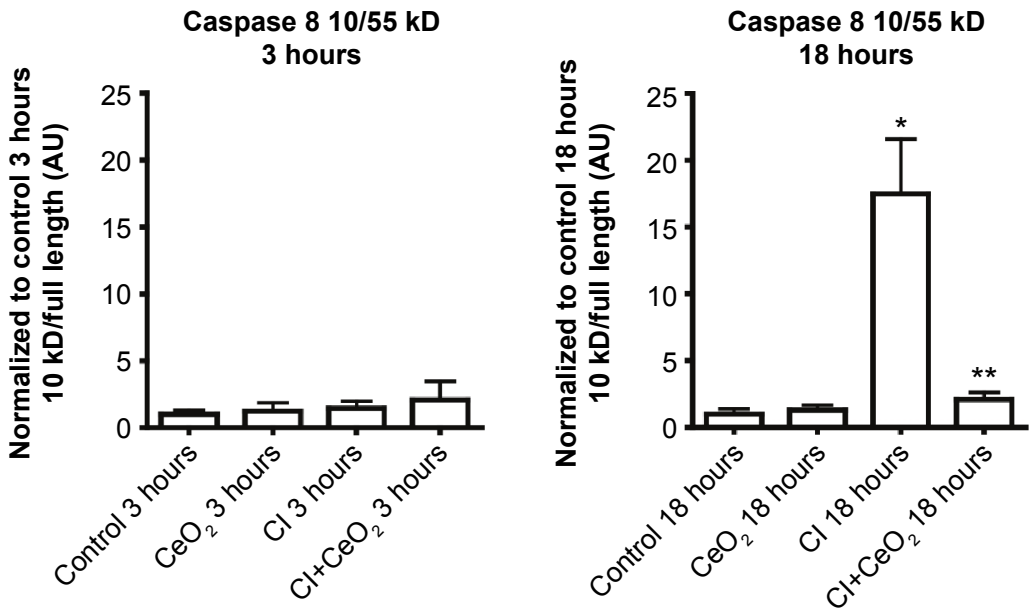

Figure 6 Cerium oxide $\left(\mathrm{CeO}_{2}\right)$ nanoparticle treatment decreases protein ubiquitination and caspase 8 cleavage during sepsis.

Notes: Effect of nanoparticle on protein synthesis signaling was determined by immunoblot. Sepsis and treatment associated changes in protein ubiquitination (A) and caspase 8 cleavage (B). $n=6$ rats/each group, *vs Control: **vs Cl: $P<0.05$ by one-way ANOVA. 
may also be associated with diminished contractile function. ${ }^{41}$ It is thought that protein degradation is controlled, at least in part, by the activity of the muscle E3 ligases, MURF-1 and MAFbx, which, in turn, are thought to be regulated by FOXO-1. The activity of FOXO-1 is controlled by its phosphorylation by Akt, with increased FOXO-1 phosphorylation causing a decrease in its transcriptional activity. ${ }^{42}$ We found that total FOXO-1 expression was increased in CI peritonitis groups (Figure 5B). This finding is in agreement with previous reports that suggested that both cecal ligation and puncture (CLP)- and lipopolysaccharide (LPS)-induced sepsis models are associated with increased skeletal muscle FOXO-1 mRNA ${ }^{43,44}$ and protein levels. ${ }^{45}$ Conversely, we noted that peritonitis was associated with diminished ratio of Akt and FOXO-1 phosphorylated (active)/total protein and that this ratio that appeared to be attenuated with the treatment (Figure 5A and B). These data are consistent with the possibility that the $\mathrm{CeO}_{2}$ nanoparticle intervention might function to diminish the degree of sepsis-induced muscle breakdown. Why sepsis might increase total FOXO-1 expression is currently unclear, but it is possible that this change could be a compensatory mechanism to counteract sepsis-associated decreases in FOXO-1 activity. Further studies will be required to address the mechanism(s) responsible for this change, and perhaps, more importantly, the physiological relevance of why sepsis might affect FOXO-1 protein levels in skeletal muscle. In addition to its ability to protect the muscle against apoptosis and protein degradation, it is also known that septic insults cause decrease protein translation signaling. ${ }^{46,47}$ To investigate this possibility, we next determined the effect of sepsis and the nanoparticle intervention on the phosphorylation of 4EBP-1. It is thought that 4EBP-1 functions to inhibit translation by binding to the translation initiation factor elF4E. Consistent with previous work from other groups showing both endotoxemia and polymicrobial sepsis models decrease phosphorylation of 4EBP-1 in rat skeletal muscle, ${ }^{46,47}$ we found that sepsis was associated with decreased phosphorylation of 4EBP-1 (Figure 5C). Importantly, we also found that the nanoparticle treatment normalized this decreased phosphorylation of 4EBP-1 in diaphragm.

Similar to that observed for the effects of protein degradation on muscle function, it is also thought that increased muscle apoptosis can also diminish muscle function. Similar to previous reports using the LPS endotoxemia model ${ }^{48}$ and other work from our laboratory examining the effects of sepsis on circulating cytokine levels, ${ }^{15}$ we also found that this model was associated with increased caspase- 8 cleavage (Figure 6B). To determine whether increases in caspase- 8 cleavage led to increased muscle apoptosis, we next examined if peritonitis was associated with increased cleavage of caspase-3. Unlike previous data showing that LPS challenge increased caspase-3 activity in the rat diaphragm, we failed to find any change caspase-3 cleavage or caspase-3 activity in CI peritonitis model (data not shown). Supporting these data, we also failed to find any change in the ratio of Bax/Bcl-2 or in the amount of Apoptosis inducing factor (AIF) protein. Why endotoxemia but not the sepsis induced by peritonitis may be associated with increased caspase- 3 activation is currently unclear.

\section{Conclusion}

In conclusion, we have demonstrated that $\mathrm{CeO}_{2}$ nanoparticle treatment attenuated diaphragm dysfunction in a laboratory rat model of sepsis. We believe that this may be the first study to demonstrate that the systemic delivery of nanoparticles can be used to improve muscle function in a preclinical animal model of sepsis. How the nanoparticle treatment improved diaphragm contractility is currently unclear and will require additional experimentation.

\section{Acknowledgments}

This work was supported by DOE (DE-SC0005162) to ERB. The authors would like to acknowledge Niraj Nepal and Geeta Nandyala for sample collection and their technical assistance. The abstract of this article was presented at the Experimental Biology 2015 as a poster presentation with interim findings. The poster's abstract was published in FASEB journal. Asano et al (2015) Cerium oxide $\left(\mathrm{CeO}_{2}\right)$ nanoparticle treatment ameliorates peritonitis induced diaphragm dysfunction. The actual article, however, has never been published.

\section{Disclosure}

The authors declare that there are no conflicts of interests regarding the publication of this article.

\section{References}

1. Das S, Dowding JM, Klump KE, McGinnis JF, Self W, Seal S. Cerium oxide nanoparticles: applications and prospects in nanomedicine. Nanomedicine. 2013;8(9):1483-1508.

2. Nicolini V, Gambuzzi E, Malavasi G, et al. Evidence of catalase mimetic activity in $\mathrm{Ce}(3+) / \mathrm{Ce}(4+)$ doped bioactive glasses. J Phys Chem B. 2015; 119(10):4009-4019.

3. Babu KS, Anandkumar M, Tsai TY, Kao TH, Inbaraj BS, Chen BH. Cytotoxicity and antibacterial activity of gold-supported cerium oxide nanoparticles. Int J Nanomed. 2014;9:5515-5531.

4. Hirst SM, Karakoti A, Singh S, et al. Bio-distribution and in vivo antioxidant effects of cerium oxide nanoparticles in mice. Environ Toxicol. 2013;28(2):107-118.

5. Wang Q, Perez JM, Webster TJ. Inhibited growth of Pseudomonas aeruginosa by dextran- and polyacrylic acid-coated ceria nanoparticles. Int J Nanomed. 2013;8:3395-3399. 
6. Wason MS, Zhao J. Cerium oxide nanoparticles: potential applications for cancer and other diseases. Am J Transl Res. 2013;5(2):126-131.

7. Alili L, Sack M, von Montfort C, et al. Downregulation of tumor growth and invasion by redox-active nanoparticles. Antioxid Redox Signal. 2013;19(8):765-778.

8. Hirst SM, Karakoti AS, Tyler RD, Sriranganathan N, Seal S, Reilly CM. Anti-inflammatory properties of cerium oxide nanoparticles. Small. 2009;5(24):2848-2856.

9. Kong L, Cai X, Zhou X, et al. Nanoceria extend photoreceptor cell lifespan in tubby mice by modulation of apoptosis/survival signaling pathways. Neurobiol. Dis. 2011;42(3):514-523.

10. Niu J, Azfer A, Rogers LM, Wang X, Kolattukudy PE. Cardioprotective effects of cerium oxide nanoparticles in a transgenic murine model of cardiomyopathy. Cardiovasc Res. 2007;73(3):549-559.

11. Schoenberg MH, Weiss M, Radermacher P. Outcome of patients with sepsis and septic shock after ICU treatment. Langenbecks Arch Surg. 1998;383(1):44-48.

12. Aubier M, Trippenbach T, Roussos C. Respiratory muscle fatigue during cardiogenic shock. J Appl Physiol Respir Environ Exerc Physiol. 1981;51(2):499-508.

13. Hussain SN, Roussos C. Distribution of respiratory muscle and organ blood flow during endotoxic shock in dogs. J Appl Physiol. 1985;59(6): 1802-1808.

14. Scharf SM, Bark H. Function of canine diaphragm with hypovolemic shock and beta-adrenergic blockade. J Appl Physiol Respir Environ Exerc Physiol. 1984;56(3):648-655.

15. Selvaraj V, Manne ND, Arvapalli R, et al. Effect of cerium oxide nanoparticles on sepsis induced mortality and NF-kappaB signaling in cultured macrophages. Nanomedicine. 2015;10(8):1275-1288.

16. Li X, Moody MR, Engel D, et al. Cardiac-specific overexpression of tumor necrosis factor-alpha causes oxidative stress and contractile dysfunction in mouse diaphragm. Circulation. 2000;102(14):1690-1696.

17. Khawli FA, Reid MB. N-acetylcysteine depresses contractile function and inhibits fatigue of diaphragm in vitro. J Appl Physiol. 1994;77(1): 317-324.

18. Shindoh C, Hida W, Ohkawara Y, et al. TNF-alpha mRNA expression in diaphragm muscle after endotoxin administration. Am J Respir Crit Care Med. 1995;152(5 Pt 1):1690-1696.

19. Institute for Laboratory Animal Research. Guide for the Care and Use of Laboratory Animals. 8th ed. Washington (DC): National Academies Press; 2011.

20. Sharma AC, Motew SJ, Farias S, et al. Sepsis alters myocardial and plasma concentrations of endothelin and nitric oxide in rats. $J$ Mol Cell Cardiol. 1997;29(5):1469-1477.

21. Selvaraj V, Nepal N, Rogers S, et al. Inhibition of MAP kinase/ NF-kB mediated signaling and attenuation of lipopolysaccharide induced severe sepsis by cerium oxide nanoparticles. Biomaterials. 2015;59:160-171.

22. Kolli MB, Manne ND, Para R, et al. Cerium oxide nanoparticles attenuate monocrotaline induced right ventricular hypertrophy following pulmonary hypertension. Biomaterials. 2014;35(37):9951-9962.

23. Krause KM, Moody MR, Andrade FH, et al. Peritonitis causes diaphragm weakness in rats. Am J Respir Crit Care Med. 1998;157(4 Pt 1): 1277-1282.

24. Nethery D, DiMarco A, Stofan D, Supinski G. Sepsis increases contraction-related generation of reactive oxygen species in the diaphragm. J Appl Physiol. 1999;87(4):1279-1286.

25. Close RI. Dynamic properties of mammalian skeletal muscles. Physiol Rev. 1972;52(1):129-197.

26. Katta A, Thulluri S, Manne ND, et al. Overload induced heat shock proteins (HSPs), MAPKandmiRNA (miR-1 andmiR133a)response in insulin-resistant skeletal muscle. Cell Physiol Biochem. 2013;31(2-3):219-229.

27. Hayot M, Barreiro E, Perez A, Czaika G, Comtois AS, Grassino AE. Morphological and functional recovery from diaphragm injury: an in vivo rat diaphragm injury. $J$ Appl Physiol. 2001;90(6):2269-2278.

28. Petrof BJ, Shrager JB, Stedman HH, Kelly AM, Sweeney HL. Dystrophin protects the sarcolemma from stresses developed during muscle contraction. Proc Natl Acad Sci U S A. 1993;90(8):3710-3714.
29. Callahan LA, Supinski GS. Sepsis induces diaphragm electron transport chain dysfunction and protein depletion. Am J Respir Crit Care Med. 2005;172(7):861-868.

30. el-Dwairi Q, Comtois A, Guo Y, Hussain SN. Endotoxin-induced skeletal muscle contractile dysfunction: contribution of nitric oxide synthases. Am J Physiol. 1998;274(3 Pt 1):C770-C779.

31. Supinski GS, Wang W, Callahan LA. Caspase and calpain activation both contribute to sepsis-induced diaphragmatic weakness. $J$ Appl Physiol. 2009;107(5):1389-1396.

32. Lin MC, Ebihara S, El Dwairi Q, et al. Diaphragm sarcolemmal injury is induced by sepsis and alleviated by nitric oxide synthase inhibition. Am J Respir Crit Care Med. 1998;158(5 Pt 1):1656-1663.

33. Comtois AS, Barreiro E, Huang PL, Marette A, Perrault M, Hussain SN. Lipopolysaccharide-induced diaphragmatic contractile dysfunction and sarcolemmal injury in mice lacking the neuronal nitric oxide synthase. Am J Respir Crit Care Med. 2001;163(4):977-982.

34. Cheema IR, Hermann C, Postell S, Barnes P. Effect of chronic excess of tumour necrosis factor-alpha on contractile proteins in rat skeletal muscle. Cytobios. 2000;103(404):169-176.

35. Jiang J, Yang B, Han G, Yang M, Li S. Early administration of cisatracurium attenuates sepsis-induced diaphragm dysfunction in rats. Inflammation. 2015;38(1):305-311.

36. Stasko SA, Hardin BJ, Smith JD, Moylan JS, Reid MB. TNF signals via neuronal-type nitric oxide synthase and reactive oxygen species to depress specific force of skeletal muscle. J Appl Physiol. 2013;114(11): 1629-1636.

37. Wilcox PG, Wakai Y, Walley KR, Cooper DJ, Road J. Tumor necrosis factor alpha decreases in vivo diaphragm contractility in dogs. Am J Respir Crit Care Med. 1994;150(5 Pt 1):1368-1373.

38. Yu X, Kennedy RH, Liu SJ. JAK2/STAT3, not ERK1/2, mediates interleukin-6-induced activation of inducible nitric-oxide synthase and decrease in contractility of adult ventricular myocytes. $J$ Biol Chem. 2003;278(18):16304-16309.

39. Boczkowski J, Lanone S, Ungureanu-Longrois D, Danialou G, Fournier T, Aubier M. Induction of diaphragmatic nitric oxide synthase after endotoxin administration in rats: role on diaphragmatic contractile dysfunction. J Clin Invest. 1996;98(7):1550-1559.

40. Lee SS, Song W, Cho M, et al. Antioxidant properties of cerium oxide nanocrystals as a function of nanocrystal diameter and surface coating. ACS Nano. 2013;7(11):9693-9703.

41. Hasselgren PO, James JH, Benson DW, et al. Total and myofibrillar protein breakdown in different types of rat skeletal muscle: effects of sepsis and regulation by insulin. Metabolism. 1989;38(7):634-640.

42. Sandri M, Sandri C, Gilbert A, et al. Foxo transcription factors induce the atrophy-related ubiquitin ligase atrogin-1 and cause skeletal muscle atrophy. Cell. 2004;117(3):399-412.

43. Reed SA, Sandesara PB, Senf SM, Judge AR. Inhibition of FoxO transcriptional activity prevents muscle fiber atrophy during cachexia and induces hypertrophy. FASEB J. 2012;26(3):987-1000.

44. Schakman O, Dehoux M, Bouchuari S, et al. Role of IGF-I and the TNFalpha/ NF-kappaB pathway in the induction of muscle atrogenes by acute inflammation. Am J Physiol Endocrinol Metab. 2012;303(6):E729-E739.

45. Smith IJ, Alamdari N, O'Neal P, Gonnella P, Aversa Z, Hasselgren PO. Sepsis increases the expression and activity of the transcription factor Forkhead Box O 1 (FOXO1) in skeletal muscle by a glucocorticoiddependent mechanism. Int J Biochem Cell Biol. 2010;42(5):701-711.

46. Lang CH, Frost RA. Differential effect of sepsis on ability of leucine and IGF-I to stimulate muscle translation Initiation. Am J Physiol Endocrinol Metab. 2004;287(4):E721-E730.

47. Lang $\mathrm{CH}$, Frost RA. Endotoxin disrupts the leucine-signaling pathway involving phosphorylation of mTOR, 4E-BP1, and S6K1 in skeletal muscle. J Cell Physiol. 2005;203(1):144-155.

48. Supinski GS, Ji X, Wang W, Callahan LA. The extrinsic caspase pathway modulates endotoxin-induced diaphragm contractile dysfunction. J Appl Physiol. 2007;102(4):1649-1657. 


\section{Publish your work in this journal}

The International Journal of Nanomedicine is an international, peerreviewed journal focusing on the application of nanotechnology in diagnostics, therapeutics, and drug delivery systems throughout the biomedical field. This journal is indexed on PubMed Central, MedLine, CAS, SciSearch ${ }^{\circledR}$, Current Contents ${ }^{\circledR} /$ Clinical Medicine,

Journal Citation Reports/Science Edition, EMBase, Scopus and the Elsevier Bibliographic databases. The manuscript management system is completely online and includes a very quick and fair peer-review system, which is all easy to use. Visit http://www.dovepress.com/ testimonials.php to read real quotes from published authors.

Submit your manuscript here: http://www.dovepress.com/international-journal-of-nanomedicine-journal 